\title{
Sound quality assessment of a medium-range aircraft with enhanced fan-noise shielding design
}

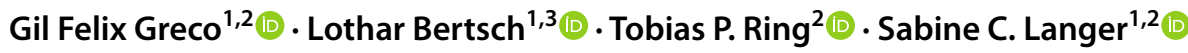

Received: 6 December 2020 / Revised: 8 March 2021 / Accepted: 31 March 2021 / Published online: 21 April 2021

(c) The Author(s) 2021

\begin{abstract}
The investigation of technologies that can improve the sustainability of the air transport system requires not only the development of alternative fuel concepts and novel vehicle technologies but also the definition of appropriate assessment strategies. Regarding noise, the assessment should reflect the situation of communities living near airports, i.e., not only addressing sound levels but also accounting for the annoyance caused by aircraft noise. For this purpose, conventional A-weighted sound pressure level metrics provide initial but limited information as the level- and frequency-dependency of the human hearing is accounted for in a simplified manner. Ideally, subjective evaluations are required to adequately quantify the perceived short-term annoyance associated with aircraft noise. However, listening tests are time-consuming and not suitable to be applied during the conceptual aircraft design stage, where a large solution space needs to be explored. Aiming at bridging this gap, this work presents a methodology for the sound quality assessment of computational aircraft noise predictions, which is hereby conducted in terms of objective psychoacoustic metrics. The proposed methodology is applied to a novel medium-range vehicle with fan noise shielding architecture during take-off and landing procedures. The relevance of individual sound sources, i.e., airframe and engine noise contributions, and their dependencies on the aircraft architecture and flight procedures are assessed in terms of loudness, sharpness, and tonality. Moreover, the methodology is steered towards community noise assessment, where the impacts on short-term annoyance brought by the novel aircraft design are analysed. The assessment is based on the modified psychoacoustic annoyance, a metric that provides a quantitative description of human annoyance as a combination of different hearing sensations. The present work is understood as an essential step towards low-annoyance aircraft design.
\end{abstract}

Keywords Aircraft noise $\cdot$ Environmental acoustics $\cdot$ Sound quality $\cdot$ Community noise $\cdot$ Short-term annoyance

\section{Introduction}

To compensate the adverse environmental impacts brought by the civil aviation, the European vision set by Flightpath 2050 [1] aims at ambitious goals: a reduction of $75 \%$ on $\mathrm{CO}_{2}$ emissions per passenger kilometer and a $65 \%$ cut of

Gil Felix Greco

g.felix-greco@tu-braunschweig.de

1 Cluster of Excellence $\mathrm{SE}^{2} \mathrm{~A}$, Sustainable and Energy-Efficient Aviation, Technische Universität Braunschweig, 38106 Braunschweig, Germany

2 Institute for Acoustics, Technische Universität Braunschweig, Langer Kamp 19, 38106 Braunschweig, Germany

3 Institute of Aerodynamics and Flow Technology, German Aerospace Center (DLR), Bunsenstraße 10, 37073 Göttingen, Germany the perceived noise levels, among others. These reductions are relative to technologies available on typical civil aircraft in the year of 2000. Aiming at the identification of gamechanging technologies that can contribute to a greener future air transport system, the German Cluster of Excellence $\mathrm{SE}^{2} \mathrm{~A}$ - Sustainable and Energy Efficient Aviation was established at TU Braunschweig in 2019 [2]. This task requires not only the investigation of alternative fuels and the development of novel vehicle technologies but also the definition of adequate assessment strategies.

Regarding noise, a comprehensive assessment should consider the situation of the exposed communities living at the vicinities of airports by addressing not only sound levels but also accounting for the annoyance caused by aircraft noise. Commonly, A-weighted sound pressure level metrics are employed to quantify the impact of a single flight event. These levels are then processed into the sound exposure 
level (SEL) metric per flight which is furthermore translated into an energy equivalent sound pressure level to describe the long-term impact of the air-traffic on communities. However, this is a coarse approach since it will account for the level- and frequency-dependency of the human hearing in a simplified manner. Furthermore, the complex temporal and spectral structure associated with the noise perceived during each fly-over event is not directly taken into account, hence the quantitative assessment does not include enough information to quantify human annoyance.

Another typically applied metric is the Effective Perceived Noise Level (EPNL), which was specifically developed in the 1960's as a predictor of human annoyance to aircraft noise [3]. It accounts for sound magnitude, spectral content, event duration and the presence of tones. However, perceptual studies conducted in the early 1990's indicated that loudness could be a better predictor of short-term annoyance than the EPNL [4]. Moreover, the EPNL tone correction procedure is often discussed in the literature regarding its capabilities to quantify subjective responses to aircraft noise that contains multiple complex tones [5, 6].

According to the goal of a noise assessment describing the situation of affected communities, psychoacoustic metrics, which are deeply based on the human auditory system, offers an alternative way to quantitatively describe human annoyance to aircraft noise. In fact, psychoacoustic metrics were recently employed by several studies as sound quality (SQ) indicators in the context of single flight events, for applications such as: aircraft conceptual design [7, 8], for the SQ characterization of aircraft under operational conditions [9, 10], and to assess noise abatement procedures [11]. Moreover, by verifying that loudness and tonality play an important role on human annoyance to aircraft noise, More [12] proposed a modified psychoacoustic annoyance model that was able to outperform the EPNL in approximating short-term annoyance ratings of jet-powered aircraft sounds.

In order to adequately evaluate the perceived annoyance associated to aircraft noise, subjective evaluations via listening tests are required. Consequently, recent activities of several research entities around the world focuses on the auralization of simulated aircraft noise with subsequent listening tests [13-15]. Although this concept is straightforward, due to it's complexity and inherent limitation regarding the number of test cases, it is not applicable to large solution spaces. Therefore, the main motivation of this work lies in the development and application of a framework for the objective sound quality evaluation of environmental aircraft noise predicted through computational simulations.

Single-aisle aircraft correspond to $34 \%$ of the total global aircraft fleet operating in the year of 2015 and are expected to compose $71 \%$ of the global fleet by 2035 [16]. Thus, the present study focuses on the application of the developed methodology to assess the sound quality of an innovative medium-range aircraft design with fan-noise shielding architecture. This vehicle was selected from the work of Bertsch [17] due to its low-noise potential, and was assessed by previous works $[18,19]$ by means of conventional sound metrics. For the sake of comparison, a reference aircraft with specifications similar to an Airbus A319 is also considered in our study.

This work is organized as follows: Sect. 2 presents the methodology applied, which is based on two steps: Sect. 2.1 presents the simulation process employed for the design synthesis and noise prediction of the aircraft, while Sect. 2.2 describes the sound quality analysis tool along with the implemented psychoacoustic metrics. Section 3 gives an overall description of the vehicle concepts and study setup considered in this work while the results are presented in Sect. 4. Finally, Sect. 5 presents the conclusions and outlook.

\section{Methodology}

The development of future low-noise aircraft concepts requires computational simulation tools capable to provide reliable noise predictions while considering realistic operational conditions. The noise prediction of the aircraft designs considered in this work (see Sect. 3) is based on the simulation process established and validated by Bertsch [17, 18]. An overall picture of this methodology is illustrated in Fig. 1, which is composed by three tools: (1) the Preliminary Aircraft Design and Optimization (PrADO) tool [20], (2) the SHADOW tool [21], which accounts for sound shielding effects, and (3) the Parametric Aircraft Noise Analysis Module (PANAM) [17]. This framework is capable to predict the noise immission levels, i.e., sound pressure level (SPL) received at arbitrary observer positions on the ground, of current and novel aircraft designs along a given flightpath. The noise immissions are then assessed in terms of standard SPL-based metrics as well as certification metrics based on the Perceived Noise Level (PNL). As a contribution of the present work, the Sound Quality Analysis Tool (SQAT) is incorporated into this framework so that the noise assessment capabilities could be further extended in terms of objective psychoacoustic metrics. In the following, a detailed description of the upgraded simulation process is given.

\subsection{System noise prediction}

The underlying system noise prediction is performed with the DLR tool PANAM [17]. This tool is comprised of so called sound source models that simulate the noise emission of specific components onboard of the aircraft during flight. The major noise sources onboard tube-and-wing aircraft with turbofan engines are accounted for. If major noise 
Fig. 1 Overall simulation process

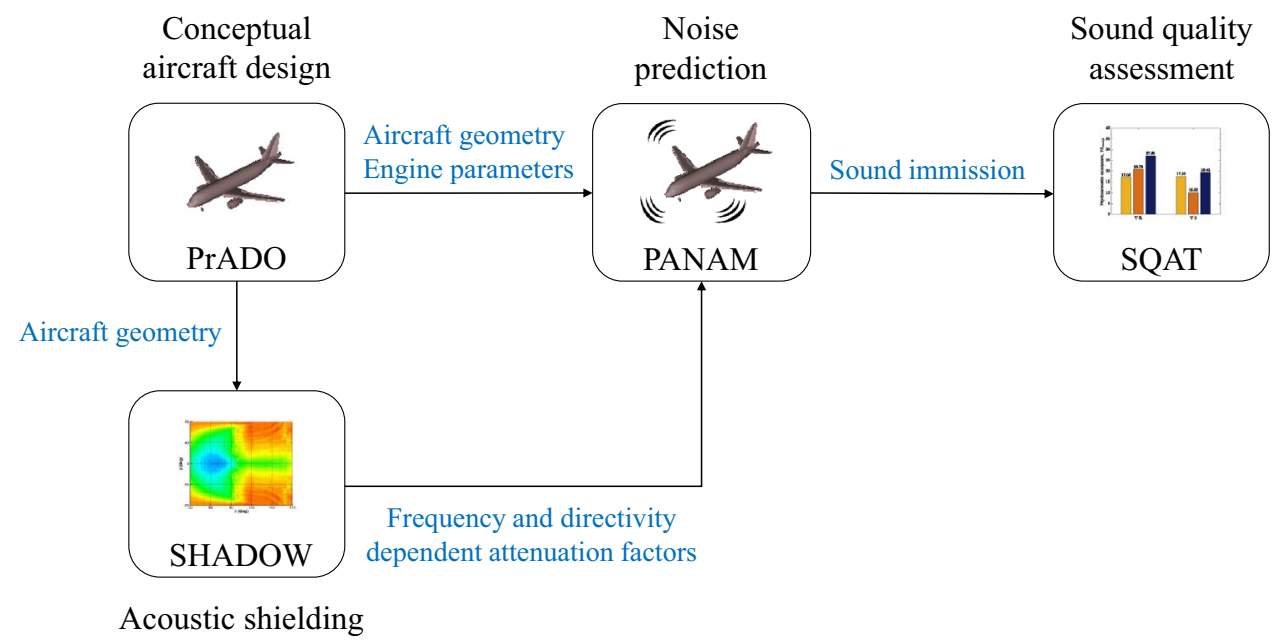

sources are adequately captured, the overall noise emission of an aircraft can be approximated, e.g., see verification of results [17] or results of tool benchmark tests [19, 22, 23]. The implemented noise source models are semi-empirical, i.e., they predict absolute emission noise levels for each modeled source according to the current operational condition of the aircraft. The models are furthermore parametric with respect to the aircraft and engine geometry meaning that different geometries and designs can be assessed within certain model-specific and defined limitations. For example, an implemented semi-empirical model for the leading edge high-lift devices can be applied to a certain design concept of this device, i.e., conventional slotted slat and Kruger systems [24]. Limitation of the design concept is caused by the semi-empirical nature of the model. Furthermore, certain detailed parameters are not subjected to a modification since they are not explicitly resolved by the model but inherently accounted for by the semi-empirical nature of the model.

The noise prediction tool PANAM accounts for the classical noise sources onboard an aircraft, i.e., airframe and engine noise sources. The source models considered in this study are summarized in Table 1 .

Acoustic shielding effects due to the positioning of the engine in relation to the airframe geometry can be accounted for in PANAM via a dedicated interface to the DLR tool SHADOW [21], which provides frequency- and directivitydependent attenuation factors according to a given airframeengine configuration. Finally, the noise emissions are predicted by PANAM given the inputs from PrADO (airframe geometry and operational conditions along a flightpath) and the attenuation factors provided by SHADOW.

The transition from emission to immission is simulated by applying standard propagation effects, i.e., geometrical spreading and atmospheric absorption, according to ISO 9613-2 [32]. Furthermore, the Doppler shift
Table 1 Summary of simulation models used within this study

\begin{tabular}{ll}
\hline Airframe noise models & \\
\hline Trailing edge devices & DLR models [17, 25-27] \\
Leading edge devices & DLR models [17, 25, 28] \\
Landing gear & DLR models [17, 29] \\
Engine noise models & \\
Fan broadband & Modified Heidmann [30] \\
Fan tonal & Modified Heidmann [30] \\
Jet & Modified Stone [31] \\
Acoustic shielding effects & \\
- & SHADOW [21] \\
Sound propagation effects & \\
- & ISO 9613-2 [32] \\
Ground reflection and attenuation & SAE AIR 1751 [33] \\
\hline
\end{tabular}

is considered in order to account for the effect associated with a moving source in respect to a static observer. Ground reflection and attenuation are considered according to the method described by SAE AIR 1751 [33], where a grass covered ground with flow resistivity of 250 $\mathrm{kg} \cdot \mathrm{s}^{-1} \cdot \mathrm{m}^{-2}$ is employed in a flat ground topography. The received sound pressure immissions predicted by PANAM can then be translated into standard SPL-based metrics, i.e., integral levels, maximum levels, or time-level histories. Individual observers can be assessed, e.g. virtual noise certification according to ICAO ANNEX 16 [34], or contour plots can be assembled. After a PANAM simulation, sufficient input data is available to compute the objective psychoacoustic metrics implemented in the new SQAT tool or even for the auralization of aircraft flyovers, as demonstrated in other studies $[15,35]$. 


\subsection{Sound quality analysis tool}

The SQAT coupling interface with PANAM allows the assessment of any individual sound sources predicted by PANAM (see Table 1) and their possible combinations. Nevertheless, the data flow illustrated in Fig. 2 is considered in the present study. The input data is provided by PANAM in terms of SPL time-histories in constant intervals of $0.5 \mathrm{~s}$. Thereby, the contributions of the airframe and engine noise to the overall aircraft noise immission are treated separately.

The engine immission is composed by three components: (1) the jet and fan noise; (2) the buzz-saw noise, and (3) the fan harmonics. The first two are broadband noise components given in $1 / 3$ octave bands while the fan harmonics are tonal noise components predicted on their actual central frequency. The information about the $i^{\text {th }}$ tone's central frequency, $f_{\mathrm{c}, i}$, and SPL, $L_{\mathrm{p}, i}$, are employed for the computation of the Aures' tonality, as it will be explained in more details in Sect. 2.2.3.

To compute the total engine immission, the tones predicted by the fan harmonics model are added to the corresponding nearest $1 / 3$ octave band from their central frequencies and then summed incoherently with the two other previously mentioned sound sources. The total immission of the aircraft is then obtained from the incoherent sum of the broadband airframe noise (provided in $1 / 3$ octave SPL time-histories) with the total engine immission levels. The objective psychoacoustic metrics implemented in SQAT are described in the following.

\subsubsection{Loudness}

Loudness is defined as the subjective perception of the magnitude of a sound, which is a function of its level, spectral content and duration. The Zwicker loudness model, which is standardized by ISO 532-1 [36], is employed in this work. The inputs for the loudness computation are unweighted SPL in $1 / 3$ octave bands from $25 \mathrm{~Hz}$ to $12.5 \mathrm{kHz}$. The loudness calculation procedure involves three main steps:

(i) The physical sound spectra is converted into excitation levels in the critical band rate, $z$, which describes the frequency resolution of the human auditory system in the Bark scale [37].

(ii) The specific loudness, $N^{\prime}(z)$, which corresponds to the loudness distribution across the 24 critical bands, is determined from the excitation levels.

(iii) The overall loudness, $N$, is obtained from the integration of the specific loudness over the whole Bark scale, as

$$
N=\int_{0}^{24 \text { Bark }} N^{\prime}(z) \mathrm{d} z \quad \text { [sone]. }
$$

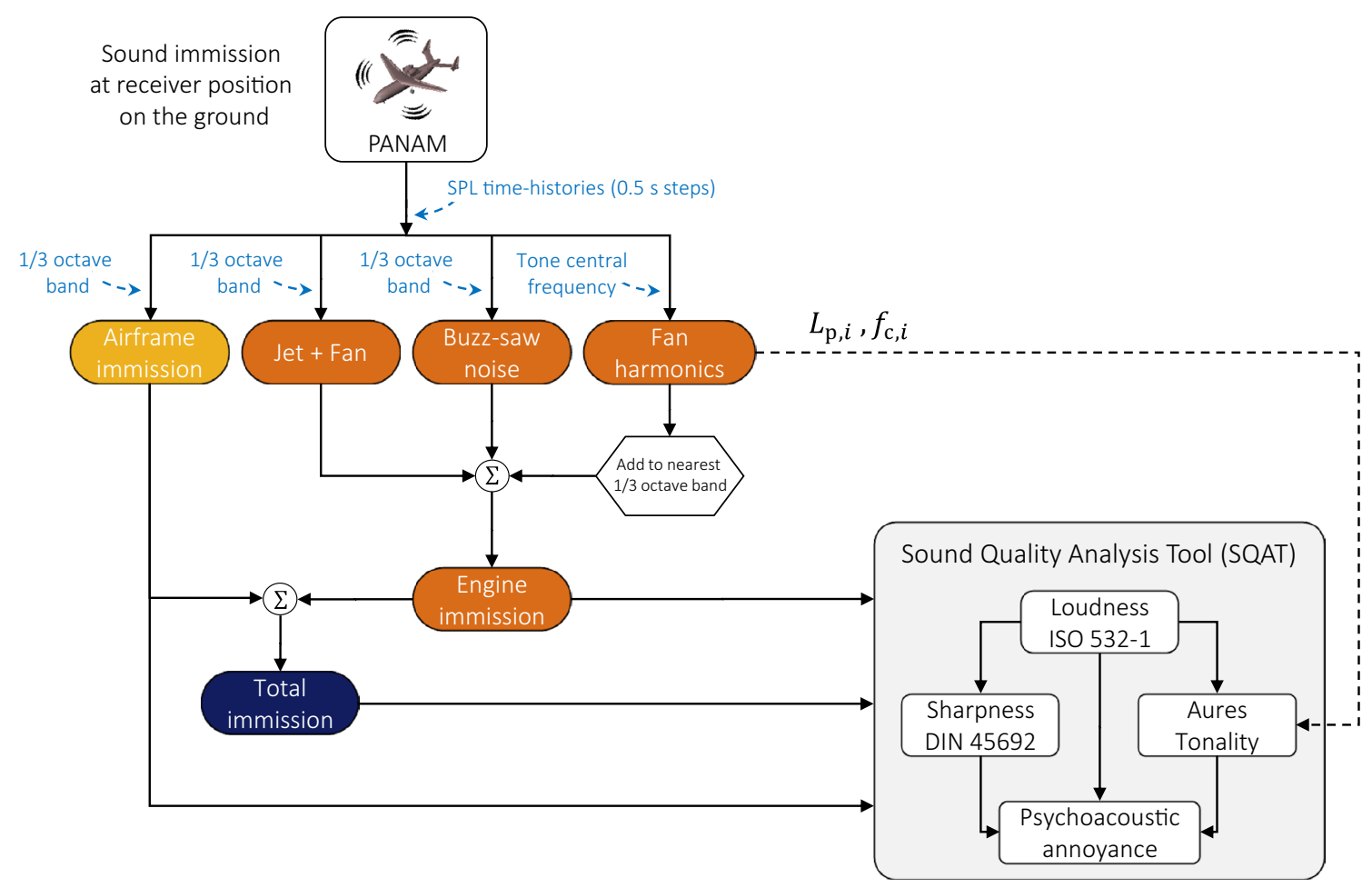

Fig. 2 Sound quality analysis tool: schematic representation of the input data flow employed in this work 
The unit of Loudness is sone, which expresses the human sensation of sound volume in a linear scale (meaning that a sound with 12 sone, for example, is perceived twice as loud as a sound with 6 sone). As a reference, a $1 \mathrm{kHz}$ narrowband tone (one critical-band wide) with a level of $40 \mathrm{dBSPL}$ has a loudness of 1 sone.

\subsubsection{Sharpness}

The sharpness, $S$, is the hearing sensation related to the amount of high-frequency content contained in the sound spectrum, which is associated to the perceived "brightness" of a sound. Sharpness is given in acum, where a narrowband noise (one critical-band wide), with a central frequency of $1 \mathrm{kHz}$ and having a level of $60 \mathrm{dBSPL}$ has a sharpness of 1 acum. The sharpness is hereby calculated according to the level-independent model described by DIN 45692 [38], as the ratio between the sound magnitude in the high frequencies and the overall sound magnitude, i.e., along the whole frequency range, which is given by

$S=0.11 \cdot \frac{\int_{0}^{24} \operatorname{Bark}^{\prime}(z) \cdot g(z) \cdot z \mathrm{~d} z}{N} \quad$ [acum].

The function $g(z)$, which is applied to weight the high-frequency content above $15.8 \mathrm{Bark}(\approx 3054 \mathrm{~Hz})$, is given by

$g(z)= \begin{cases}0.15 \cdot e^{0.42(z-15.8)}+0.85 & , z>15.8 \text { Bark } \\ 1 & , z \leq 15.8 \text { Bark }\end{cases}$

\subsubsection{Tonality}

Tonality plays an important role on human annoyance to a sound as it can be very irritating even in low amplitudes. The Aures' tonality model is employed by this work as it was verified by the works of Minard et al. [5] and More [12] to adequately predict perceived unpleasantness ratings of aircraft tonal noise.

Based on subjective evaluations of pure tones and narrowband noises, Aures [39] developed a model which could predict with a high degree of correlation how tonal a sound is perceived. The Aures' model expresses tonality, $K$, in tonal units (t.u.), where 1 t.u. corresponds to the tonality of $1 \mathrm{kHz}$ pure tone with a level of $60 \mathrm{dBSPL}$. Figure 3 illustrates the procedure implemented in SQAT for the computation of the Aures' tonality model.

The information about the central frequency, $f_{\mathrm{c}, i}$, and the SPL, $L_{\mathrm{p}, i}$, of the $i$-th tonal content of the fan harmonics are used to compute a tonal weighting, which is a function of the tone's central frequency, bandwidth and the sound pressure level excess. The reader is referred to the work of Hastings et al. [40] for a complete description on the computation of

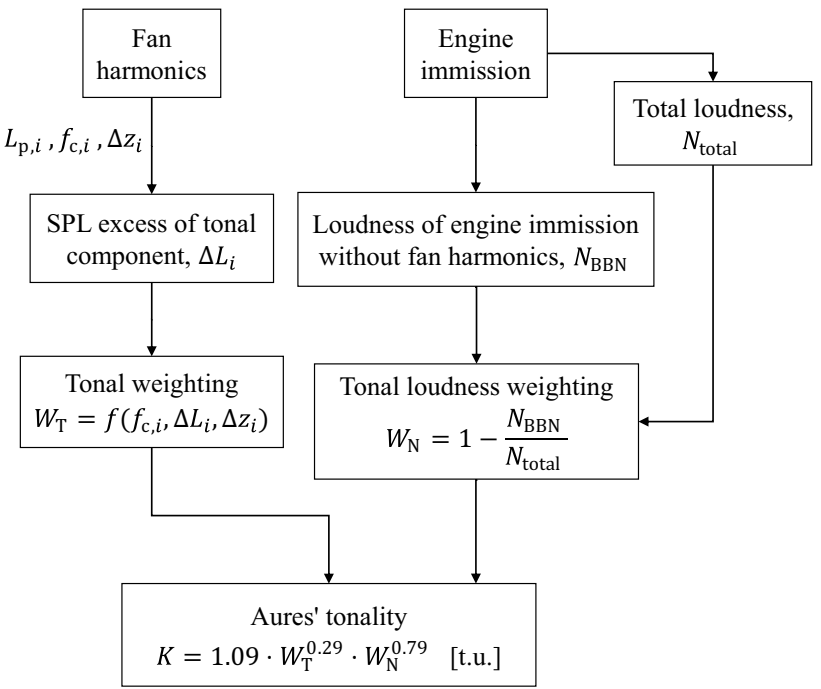

Fig. 3 Schematic of the Aures' tonality computation procedure implemented in the SQAT

the tonal weighting. The sound pressure level excess, $\Delta L_{i}$, which is employed to quantify the aural relevance of the tones, is based on the procedure proposed by Terhard et al. [41]. As PANAM does not provide any information about the bandwidth of the tones, $\Delta z_{i}$, the approach adopted by Sahai [7] is adopted in the present work, i.e., a constant percentage bandwidth of $27.5 \%$ of the critical-band around each tonal component is used. Finally, the tonality is computed by a weighted multiplication of the tonal weighting, $W_{\mathrm{T}}$, with the tonal loudness weighting, $W_{\mathrm{N}}$, which is calculated considering the ratio of the loudness of the engine immission without the tonal components, $N_{\mathrm{BBN}}$, and the loudness of the total engine immission $N_{\text {total }}$.

\subsubsection{Psychoacoustic annoyance}

Fastl and Zwicker [37] developed a metric, called psychoacoustic annoyance (PA), capable to approximate annoyance ratings obtained via jury evaluations of synthetic and everyday sounds (e.g., noise from drills, cars and air conditioners). This is achieved through the combination of different psychoacoustic metrics, which can describe the human perception of distinct hearing sensations attributed to loudness, spectral distribution, and the temporal structure of sounds. This work employs the modified version of the Zwicker's psychoacoustic annoyance, $\mathrm{PA}_{\text {mod }}$, as an indicator for the short-term annoyance associated to aircraft flyover events. The $\mathrm{PA}_{\text {mod }}$ model was developed by More [12] in the context of aircraft noise in order to incorporate the influence of tonality on the original Zwicker's psychoacoustic annoyance model. Due to limitations of the framework employed in this work for the aircraft system noise predictions, the 
terms accounting for the effect of roughness and fluctuation strength are not considered as the computation of these metrics would require a finer frequency and temporal discretization (shorter than the $0.5 \mathrm{~s}$ offered by most parametric emission models). Nevertheless, More [12] observed that the contribution of those two metrics to annoyance ratings is typically negligible for jet-powered aircraft. Thus, the version of the $\mathrm{PA}_{\text {mod }}$ employed in the present work is computed as

$\mathrm{PA}_{\text {mod }}=N\left(1+\sqrt{\gamma_{0}+\gamma_{\mathrm{S}} \omega_{\mathrm{S}}^{2}+\gamma_{\mathrm{T}} \omega_{\mathrm{T}}^{2}}\right)$,

given the constants $\gamma_{0}=-0.16, \gamma_{\mathrm{S}}=11.48$ and $\gamma_{\mathrm{T}}=1.25$. The terms $\omega_{\mathrm{S}}$ and $\omega_{\mathrm{T}}$ account for the influence of loudness, sharpness and tonality, as

$\omega_{\mathrm{S}}= \begin{cases}0.25(S-1.75) \log _{10}(N+10) & , S>1.75 \\ 0 & , S \leq 1.75\end{cases}$

and

$\omega_{\mathrm{T}}=\left(1-e^{-0.29 N}\right)\left(1-e^{-5.49 K}\right)$.

\section{Vehicle description and study setup}

For this work, two medium-range vehicles from the work of Bertsch [17] were selected: (1) a reference vehicle, hereafter referred as V-R, which has specifications similar to an Airbus A319, and (2) a novel low-noise vehicle design, denominated subsequently as V-2, with enhanced sound shielding properties promoted by the overall aircraft layout as a high-wing configuration and positioning of the engines

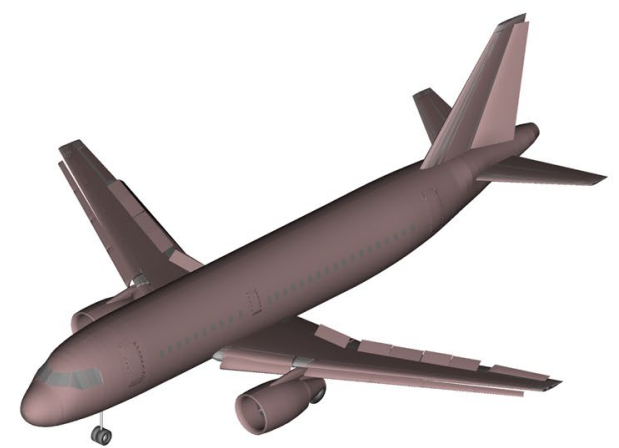

(a) Reference medium-range aircraft: V-R. above the fuselage-wing junction. The selected aircraft are illustrated in Fig. 4.

Both aircraft were designed in PrADO using specifications of a CFM56-5A5 turbofan engine, which has a bypass ratio of 6 . Besides the benefit of the fan noise shielding, conventional installation effects $[42,43]$ due to the interaction of the jet exhaust flow with the aircraft's high lift devices (HLD) are avoided. Furthermore, the V-2 design has a $\mathrm{H}$-tail in order to avoid the direct interaction of the jet exhaust with the aircraft's fin. Another difference between the aircraft designs is that the landing gear is mounted in the fuselage for the V-2 instead of under the wing, which consequently reduces its length by $35 \%$ [44]. Another consequence of the different landing gear installation is the avoidance of any gear wake impingement on extracted trailing edge high-lift devices hence preventing a potential noise source by design. A complete characterization of these two aircraft regarding environmental noise is presented in previous works [17-19, 22, 23] by means of conventional sound metrics. It is worth mentioning that the V-2 layout is probably unfavorable regarding the internal cabin noise as the engines are mounted directly on the fuselage. The possible interaction between the hydrodynamic and acoustic fields of the jet exhaust flow with the aircraft fuselage as well as the structure-borne sound transmitted from the engines to the cabin can be expected to have a negative impact on the internal noise when compared to the conventional under-thewing engine configuration.

The flight trajectories employed by both aircraft during approach and departure procedures are shown in Fig. 5. For both cases, straight trajectories aligned with the runway center are considered. The approach, which is conducted with a continuous descent glide slope of $\approx 3^{\circ}$ and the engine speed in idle, has three distinct HLD configurations: (1) until $\approx-10.7 \mathrm{~km}$ before the runway threshold (flap $15^{\circ}$ and slat $20^{\circ}$ ); (2) from $\approx-10.7 \mathrm{~km}$ to $\approx-6.6 \mathrm{~km}$ the high lift devices

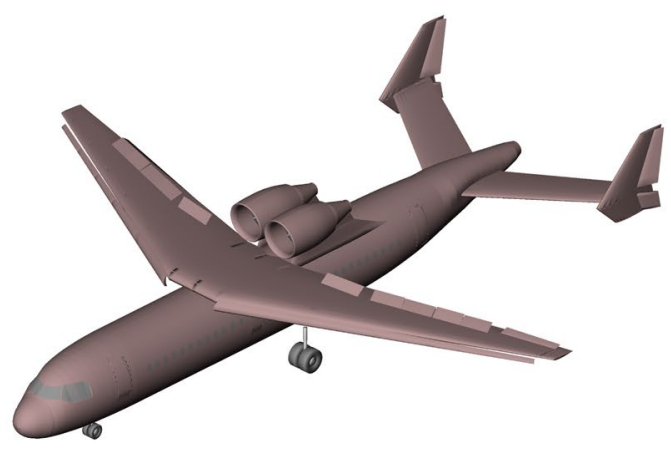

(b) Novel low-noise vehicle with fan noise shielding architecture: $\mathrm{V}-2$.

Fig. 4 Aircraft designs considered in this work 


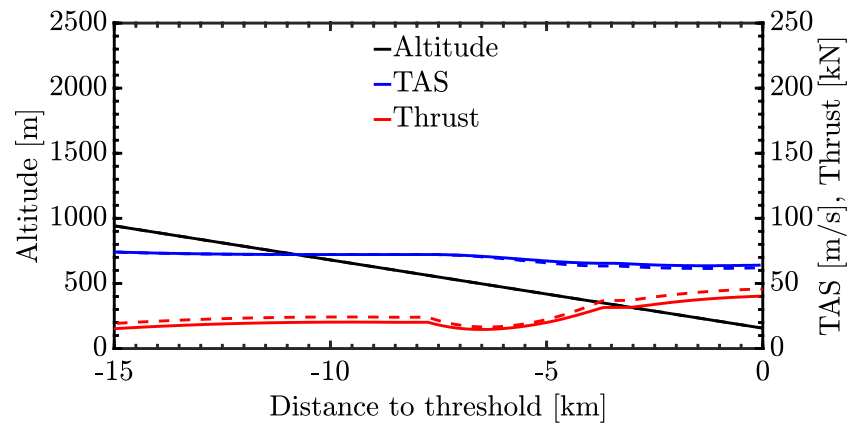

(a) Approach.

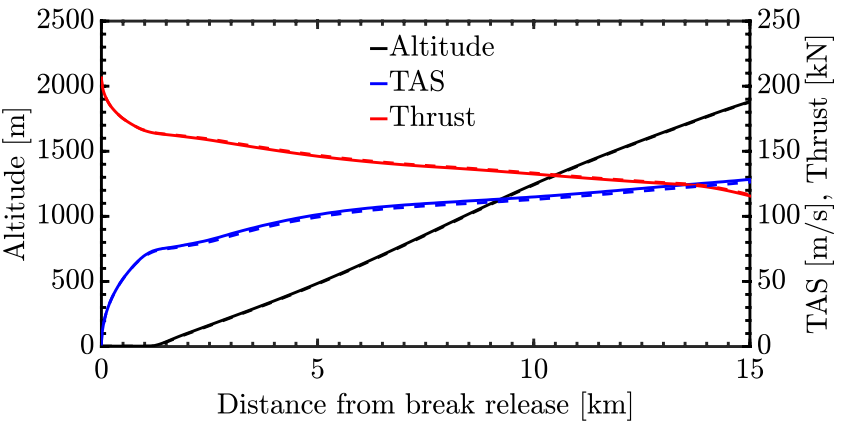

(b) Departure.

Fig. 5 Elevation profiles, engine thrust and the aircraft's true air speed (TAS) along the prescribed flight trajectories for the V-R (solid lines) and V-2 (dashed lines) vehicles

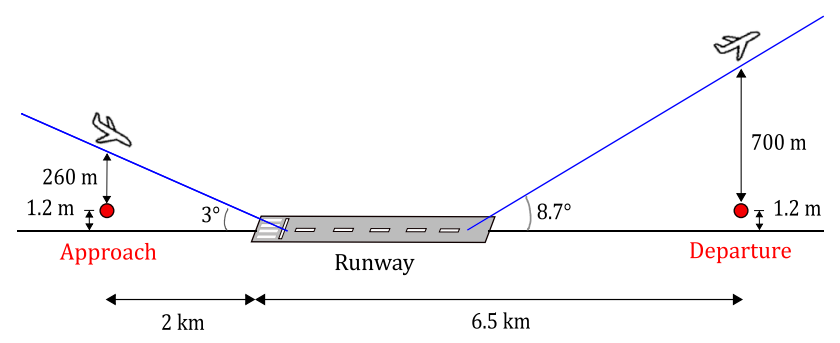

Fig. 6 Single observer positions (red circles) considered in this study. For the sake of clarity, the real proportions are not maintained

are fully deployed (flap $35^{\circ}$ and slat $25^{\circ}$ ), and (3) the landing gears are deployed from $\approx-6.6 \mathrm{~km}$ onward. Due to different aerodynamic performance of the two vehicles, more thrust is required to operate the $\mathrm{V}-2$ along the predefined approach trajectory. The departure is conducted with a constant climb angle of $\approx 8.72^{\circ}$, full thrust and engines at a rotational speed $N_{1}=100 \%$.

\subsection{Observer positions for noise assessment}

Two single observer positions (one for each flight procedure) positioned at a height of $1.2 \mathrm{~m}$ above the ground are considered (see Fig. 6). The height of the observers was chosen in order to conform with the recommendations made by ICAO Annex 16 [34]. For the approach procedure, the observer is positioned at a distance of $-2 \mathrm{~km}$ from the runway threshold (aircraft flyover altitude of $\approx 260 \mathrm{~m}$ ), while an observer positioned at $6.5 \mathrm{~km}$ from break release (aircraft flyover altitude of $\approx 700 \mathrm{~m}$ ) is considered during the departure procedure.

\section{Results and discussion}

In this section, the framework described in Sect. 2 is applied to assess the sound quality of the reference (V-R) and low-noise (V-2) vehicles during approach and departure procedures. Due to the component-wise noise modeling capability of PANAM, not only the overall aircraft noise immissions but also the contributions of the airframe and engine components are assessed and discussed in terms of sound quality metrics (SQM). The upcoming analyses are computed at the single observer positions presented previously in Sect. 3.1.

Before performing the objective assessment of the aircraft by means of SQM, the physical magnitude of the sound spectra reaching the observer positions in the ground is analysed as it provides useful insights to support the upcoming analyses. For this purpose, Fig. 7 presents the aircraft noise immission spectra in terms of equivalent sound pressure level, $L_{\mathrm{p}, \text { eq }}$, i.e., the unweighted sound spectra energy averaged over the entire flight event duration.

For the approach procedure (see Fig. 7a), the sound spectra for the reference aircraft is dominated by the airframe noise at low-frequencies below $400 \mathrm{~Hz}$ and by the engine noise for frequencies above $1 \mathrm{kHz}$. Due to the engine shielding, the contribution of the engine noise is considerably reduced toward high frequencies $(\approx 13 \mathrm{~dB}$ at $2.5 \mathrm{kHz})$ for the V-2 aircraft, while the airframe contribution remained basically unchanged.

During departure (see Fig. 7b), the engine contribution dominates the whole immission spectra for the reference vehicle. Even with the integration of the spectral energy over the entire flyover duration, which makes the $L_{\mathrm{p} \text {,eq }}$ a weak indicator to evaluate non-stationary tonal content, a prominent tone at $2.5 \mathrm{kHz}$ corresponding to the first fan harmonic is observed. The results show that V-2 architecture promotes a significant reduction of the engine noise for the entire frequency range. As a consequence, the relative contribution of the airframe noise to the overall noise immission of the $\mathrm{V}-2$ vehicle becomes relevant.

The sound immissions of the aircraft designs are assessed in terms of the proposed SQM in Fig. 8. We assume that the loudness is stationary during the 0.5 second time-interval modeled by PANAM. As the sound event of an aircraft 


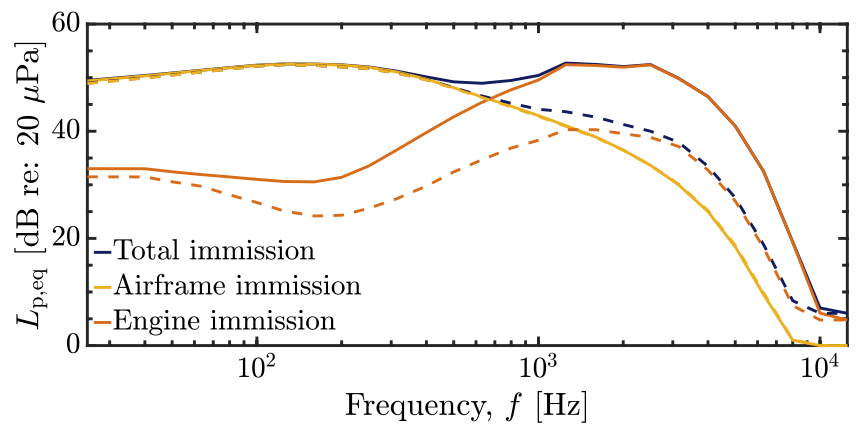

(a) Approach.

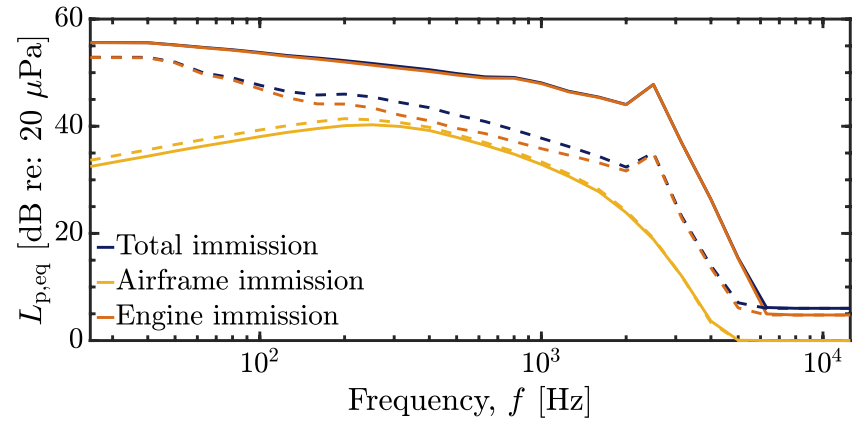

(b) Departure.

Fig. 7 Time-averaged sound immission spectra (1/3 octave bands) of the V-R (solid-lines) and V-2 (dashed-lines) aircraft

flyover perceived by an observer in the ground is non-stationary, $5 \%$ percentile values (i.e., value exceeded in $5 \%$ of the entire flyover event duration) are employed to quantify loudness, following the recommendation of ISO 532-1 [36] for the analysis of time-varying sounds. The same criteria is also adopted for the other SQM being considered in our study due to the lack of recommendations.

As a general trend observed in the loudness results (see Fig. 8a/b left column), for the V-R vehicle the engine noise is perceived louder than the airframe noise during both flight procedures. During approach, this happens mainly because of the frequency-dependency of the human hearing, which penalizes frequencies below $1 \mathrm{kHz}$ (where, according to Fig. 7a, airframe noise is more relevant). During departure, it is expected that the engine noise predominates over the airframe noise for both aircraft designs as the engine is set to full power, a fact which is verified in Fig. $7 \mathrm{~b}$. With the reduction promoted by the engine noise shielding, the loudness of the airframe noise during approach becomes higher than the engine loudness for the V-2 vehicle. This is due to the fact that the airframe contribution has more energy in the frequency range where the human hearing is more sensitive (around $1 \mathrm{kHz}$ ) whereas the engine contribution is verified to be more prominent at low frequencies, as can be verified in Figure $7 \mathrm{~b}$. Overall, the total immission loudness of the V-2 vehicle during approach presents a reduction of 7.58 sone in relation to the reference aircraft, which means that the $\mathrm{V}-2$ would be perceived as $\approx 30 \%$ quieter. As the contribution of the airframe remained unchanged, further loudness reductions of the V-2 total immission during approach could be achieved by the use of low-noise airframe technologies [45]. During departure, the loudness reduction promoted by the shielding of the engine makes the V-2 airplane $\approx 48 \%$ (almost two times) quieter than the reference aircraft.

The sharpness results (see Fig. 8a/b center column) gives another perspective about the noise reductions achieved by the V-2 vehicle. During approach, when the engine noise contribution is mostly composed of high-frequency content, the shielding promoted by the $\mathrm{V}-2$ architecture contributes to a sharpness decrease of $\approx 23 \%$ for the engine noise. This reflects upon the overall vehicle sharpness, which is reduced by $\approx 21 \%$. During departure, the engine noise is not mainly composed by high-frequency noise from the fan, but also of a relevant low-frequency noise share from the jet exhaust (see Fig. 7). This fact leads to lower engine sharpness values during departure than those apparent during approach procedure. Nevertheless, a reduction of $\approx 16 \%$ is observed in the overall immission sharpness of the $\mathrm{V}-2$ vehicle during departure due to the reduction of the engine noise at high frequencies.

As a limitation of the system noise prediction process employed in this work (see Sect. 2), no information about tonal components related to the airframe is available. This is indicated by the missing bars in Fig. 8a/b, right column. Main sources of tonal components during approach are the landing gear $[46,47]$ and aeroacoustic cavity tones originated at fuel overpressure vents located in the lower wing surfaces, as observed in aircraft from the A320 family [48]. Thus, one could argue that the process adopted by the present study works with the assumption that these additional tonal sources are removed, e.g., by the application of a vortex generator, or are assumed to be insignificant. During approach, the results for the V-R vehicle obtained for the engine noise tonality are in good agreement with the values obtained by Merino-Martinez et al. [9] for similar aircraft (A320) through experimental measurements. Hence, the tonality evaluation of the engine noise during approach using the present methodology is assumed to be representative. However, the overall aircraft tonality is underestimated if compared to those measured by Merino-Martinez et al. [9], probably due to the lack of information about the tonal components from the aircraft landing-gear. Our analysis shows that the V-2 vehicle, even with the noise shielding promoted by the engine position above the aircraft fuselage, promoted no significant tonality reductions when compared to the reference vehicle during approach. This doesn't means that 

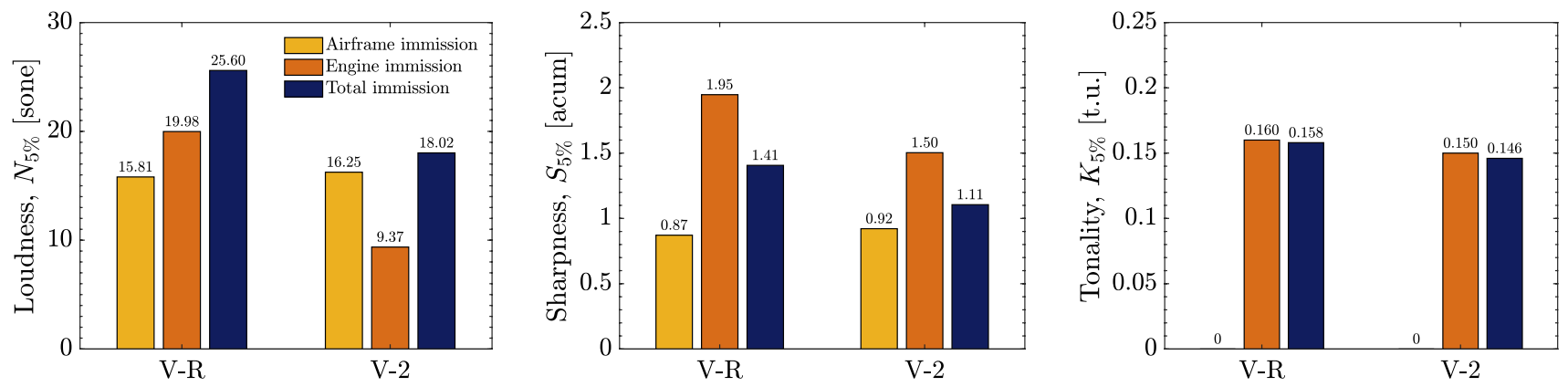

(a) Approach.
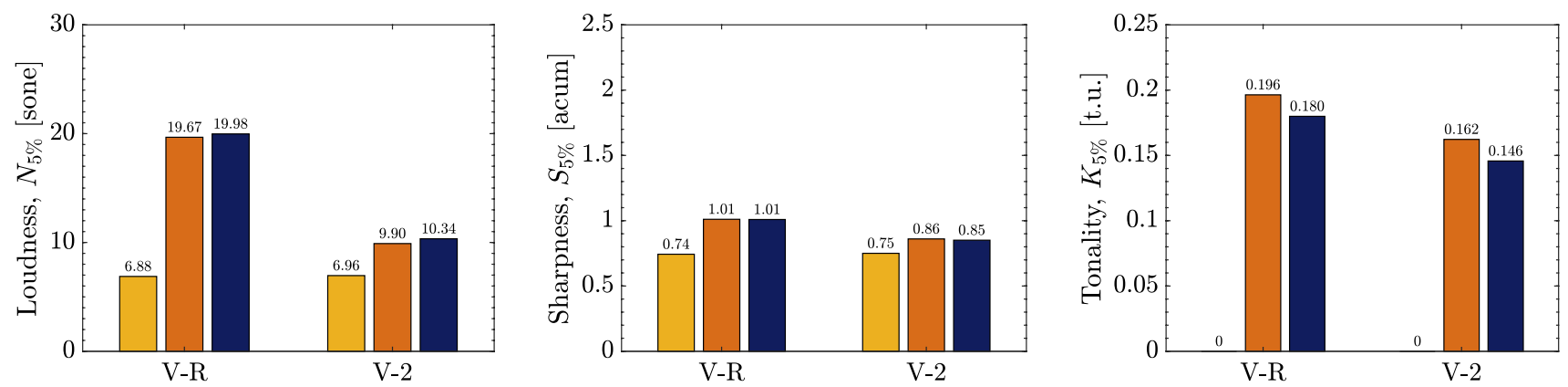

(b) Departure.

Fig. 8 Aircraft noise immission breakdown in terms of different sound quality indicators: loudness (left column), sharpness (center column) and tonality (right column). The sound quality metrics are expressed in terms of $5 \%$ percentile values

the physical amplitude of the fan tones were not decreased but rather indicates that the magnitudes of the tones and of the broadband noise (which masks the tones' perception) remained almost unaltered in relation to each other. A different picture is observed during departure, where a tonality improvement of $\approx 19 \%$ is verified for the overall V-2 aircraft immission. One last remark about the tonality analysis conducted here is that the tonality of the overall contribution is always lower than the values computed for the engine contribution. This happens because the total immission has more broadband noise contributions (airframe + engine) to mask the tones' perception than the engine noise immission.

Figure 9 provides a comparison of the total immission reduction promoted by the V-2 vehicle in terms of A-weighted conventional metrics and annoyance-based metrics. Even though using different approaches, all the selected metrics for this analysis take into account the frequencydependency of the human hearing. For the sake of comparison, the results are computed in relative percentage values, meaning that positive values imply a reduction with respect to the reference aircraft's noise immissions.

As a general trend, larger improvements during departure procedure are observed by all metrics presented in Fig. 9, which is likely due to the shielding of the engine above the fuselage since the airframe noise contribution was verified previously to remain almost unaltered during both approach and departure procedures. From the left to the right, the sound metrics presented in Fig. 9 differ substantially regarding the degree of sophistication employed to approximate the human perception to sound. Noise reductions of $12 \%$ and $15.5 \%$ for approach and departure procedures, respectively, are obtained by the $L_{\mathrm{p}, \mathrm{A}, \max }$, the simplest metric which expresses the maximum sound level magnitude occurred during the event without accounting for it's time duration. Taking into account the event duration leads to lower reductions of $8.1 \%$ and $12 \%$ for approach and departure, respectively, as observed for the sound exposure level, $L_{\mathrm{AE}}$ (also referred to as SEL). The EPNL metric, which takes

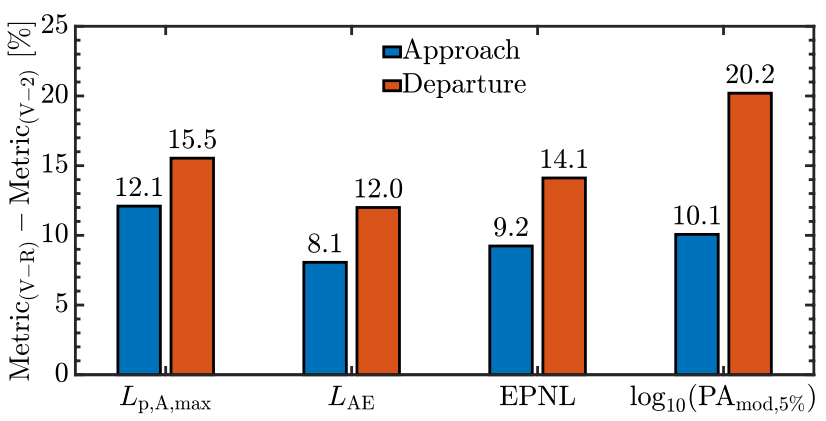

Fig. 9 Reduction of the overall aircraft immission noise in terms of conventional and annoyance-based sound metrics 
into account sound intensity, spectral content, event-duration and applies penalties to prominent tones, shows reduction values of $9.2 \%$ and $14.1 \%$.

For the sake of comparison, the modified psychoacoustic annoyance is expressed in logarithmic scale in Fig. 9. When compared to the results obtained by the EPNL, which is a similar annoyance-based metric, the $\mathrm{PA}_{\text {mod.5\% }}$ presented a similar relative reduction during approach procedure, however, significantly higher values were obtained during departure. As loudness is the main contributor to the $\mathrm{PA}_{\text {mod }}$ model, the annoyance reductions observed are mainly due to the loudness reduction of $\approx 30 \%$ and $\approx 48 \%$ promoted by the V-2 aircraft architecture during approach and departure (see Fig. 8a/b left column), respectively. These results emphasize the necessity to further investigate if short-term annoyance to aircraft noise can be better approximated by the $\mathrm{PA}_{\text {mod }}$ model than by the standard metric used for aircraft noise certification purposes, i.e., the EPNL.

In order to have a better picture on the spatial variation of the annoyance reductions promoted by the V-2 vehicle, Fig. 10 presents contours of the $\mathrm{PA}_{\text {mod,5\% }}$ for the reference aircraft (solid-lines), superimposed to (color) contours of $\mathrm{PA}_{\text {mod,5\% }}$ reduction in respect to the reference aircraft. For this analysis, a grid of receivers positioned $1.2 \mathrm{~m}$ above the ground along a longitudinal distance of $15 \mathrm{~km}$ from the runway threshold and a lateral distance of $6 \mathrm{~km}$ was considered.

During approach (see Fig. 10a), short-term annoyance reductions between 10 and $35 \%$ along the area below the flight-path can be observed. However, the short-term annoyance promoted by the V-2 aircraft exceeds that of the reference vehicle by about $10 \%$ along the lateral areas adjacent to the flight-path.

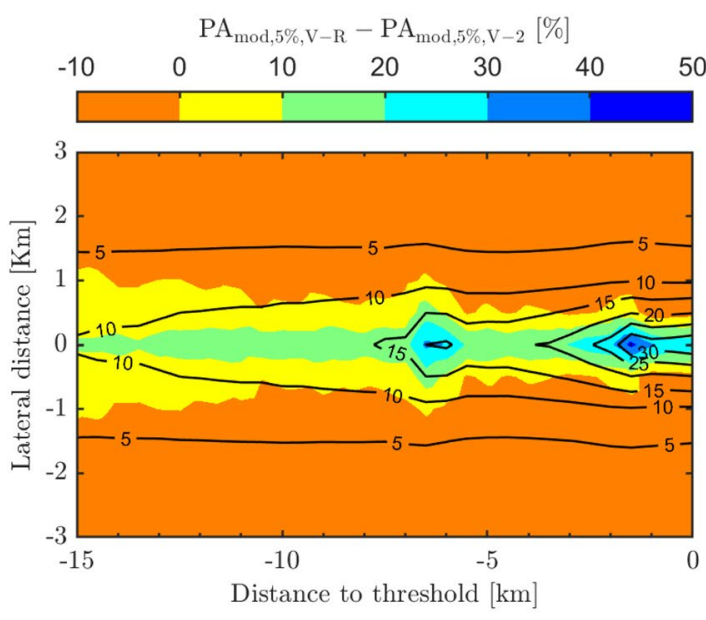

(a) Approach

Fig. 10 Short-term annoyance predictions for the overall aircraft noise immissions: contours of modified psychoacoustic annoyance (5\% percentile values) for (1) the reference aircraft (solid black lines), and (2) reduction of the V-2 vehicle with respect to the ref-
During the departure procedure, a similar trend can be verified in Fig. 10b. The fan-noise shielding design of the $\mathrm{V}$-2 aircraft has a pronounced effect in the area immediately below the flight-path, where $\mathrm{PA}_{\text {mod,5\% }}$ reductions up to $50 \%$ are verified. During departure, the flight altitude highly increases with the aircraft distance from the runway, and the $\mathrm{PA}_{\text {mod,5\% }}$ reduction becomes gradually positive for the lateral areas. The increased annoyance in the lateral areas adjacent to the flight-path is due to the combination of increased thrust and lateral directivity of the engine noise in relation to the reference aircraft. The reader is referred to the work of Bertsch [17] for more details about the directivity of the $\mathrm{V}-\mathrm{R}$ and V-2 vehicle's sound sources.

Finally, since EPNL and the modified psychoacoustic annoyance metrics are both intended to quantify human annoyance, it is of interest to investigate the degree of correlation between them. Figure 11 shows a linear regression between the results obtained by the two metrics in all the observer positions being considered in Fig. 10, for both aircraft and flight procedures, summing a total of 868 results.

The results presented in Fig. 11 show that the modified psychoacoustic annoyance model can describe $94 \%$ of the variance seen in EPNL results. The differences mainly appear due to results obtained during departure procedure, where the EPNL presented higher results than the $\mathrm{PA}_{\text {mod,5\% }}$ for some observer positions. As a remark, if only the approach procedures were considered, a correlation of $99 \%$ would be verified. Possible reasons for this discrepancy are: (1) different criteria to account for the event duration and the human perception of loudness; (2) different approaches to account for tonality, (3) the fact that only the $\mathrm{PA}_{\text {mod }}$ accounts for the influence of sharpness, and (4)

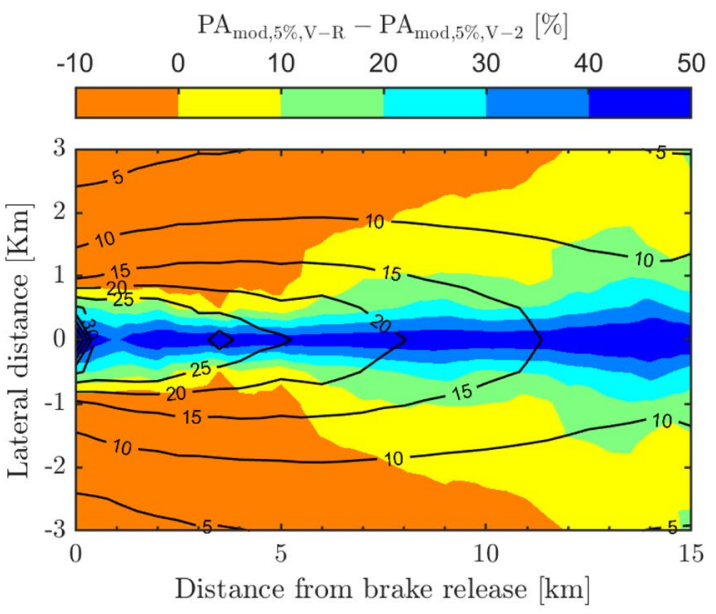

(b) Departure.

erence aircraft (colored contours), i.e., negative reduction values implies that the V-2 aircraft promotes higher short-term annoyance than the reference vehicle 


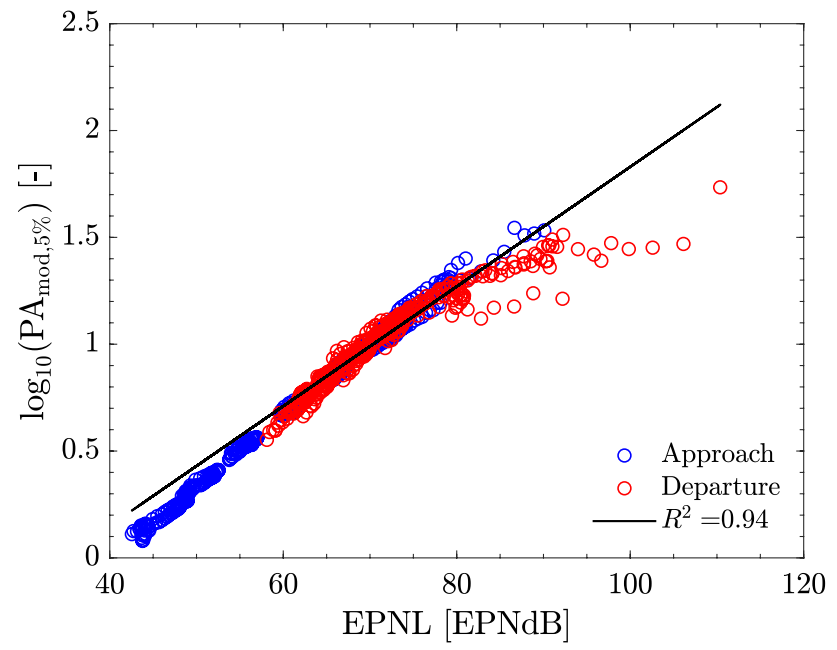

Fig. 11 Correlation between the modified psychoacoustic annoyance and EPNL metrics

neglecting the terms that account for the effect of roughness and fluctuation strength on the original modified psychoacoustic annoyance model. Nevertheless, the remarkable correlation verified in Fig. 11 suggests that, if the EPNL is able to approximate human annoyance to aircraft noise, then the $\mathrm{PA}_{\text {mod }}$ model could be considered as an alternative metric for aircraft noise certification. However, even though More [12] has already shown that the $\mathrm{PA}_{\text {mod }}$ outperforms the EPNL in approximating short-term annoyance ratings of jet-driven aircraft noise, additional investigations through perceptual tests are required in order to verify if the $\mathrm{PA}_{\text {mod }}$ is also applicable to propeller-driven aircraft sounds, as those were not considered during its development.

\section{Conclusions and outlook}

In this work, the environmental noise impact of a novel medium-range aircraft design with a high-wing layout and enhanced sound shielding properties due to the positioning of the engines above the fuselage is assessed in terms of objective psychoacoustic metrics. For this purpose, a methodology based on (1) computational predictions of aircraft noise immission using the DLR tool PANAM, and (2) a sound quality analysis tool is established. A comprehensive description of the overall process is given, including the data flow required for the computation of the psychoacoustic metrics implemented in the Sound Quality Analysis Tool (SQAT).

By means of the psychoacoustic metrics it is possible to evaluate how the sound quality of the new aircraft design differs from the reference vehicle. Firstly, the assessment comprises single observer positions directly located below the aircraft's flight-path. The analysis considering the $\mathrm{PA}_{\bmod }$ model (in logarithmic scale) shows that the shielding promoted by the engine position leads to short-term annoyance reductions of the overall aircraft immission up to $10.1 \%$ and $20.2 \%$ for approach and departure procedures, respectively. This is due to the significant loudness reduction of the engine noise immission as almost no changes related to the airframe noise contribution was verified.

Secondly, a larger assessment area is considered in order to verify the spatial distribution of the short-term annoyance reductions promoted by the V-2 aircraft. As a general trend, it is observed that the fan-noise shielding vehicle architecture studied here is beneficial for the short-term annoyance in the area directly below the flight trajectory. For lateral areas adjacent to the flight-path, an increase of the shortterm annoyance is observed mainly due to the combination of two factors: (1) the directivity of the V-2 aircraft engine noise with respect to the V-R aircraft which is increased laterally in the opposite direction from that of the flightpath, and (2) the increased thrust of the V-2 aircraft on the chosen flight-path in comparison to the reference aircraft. In general, the analysis conducted in Fig. 10 shows that the $\mathrm{PA}_{\text {mod }}$ model can be used as an indicator to assess short-term community noise annoyance.

Finally, a correlation analysis between the results obtained from EPNL and the modified psychoacoustic annoyance metrics shows a correlation of $94 \%$ between both metrics. This indicates that both metrics, the EPNL and the modified psychoacoustic annoyance, seem to (almost) equally quantify short-term annoyance to aircraft noise. Investigations on which metric is able to better describe the perceived short-term annoyance to aircraft noise or how they could be improved for this purpose are left for future works.

The present work serves as a basis for the sound quality assessment of novel aircraft designs and flight procedures being developed in the frame of the Cluster of Excellence $\mathrm{SE}^{2} \mathrm{~A}$ - Sustainable and Energy Efficient Aviation. Future work will focus on automated sound quality evaluation within large conceptual aircraft studies, aiming at (1) assessing possible improvements on current aircraft configurations, and (2) investigating novel low-annoyance aircraft designs.

Acknowledgements We gratefully acknowledge the funding by the Deutsche Forschungsgemeinschaft (DFG, German Research Foundation) under Germany's Excellence Strategy - EXC 2163/1-Sustainable and Energy Efficient Aviation-Project-ID 390881007. We acknowledge support by the German Research Foundation and the Open Access Publication Funds of Technische Universität Braunschweig. The authors would like to thank the reviewers of this paper for their valuable comments and suggestions.

Funding Open Access funding enabled and organized by Projekt DEAL. 


\section{Declarations}

Conflict of interest The authors declare that they have no conflict of interest.

Open Access This article is licensed under a Creative Commons Attribution 4.0 International License, which permits use, sharing, adaptation, distribution and reproduction in any medium or format, as long as you give appropriate credit to the original author(s) and the source, provide a link to the Creative Commons licence, and indicate if changes were made. The images or other third party material in this article are included in the article's Creative Commons licence, unless indicated otherwise in a credit line to the material. If material is not included in the article's Creative Commons licence and your intended use is not permitted by statutory regulation or exceeds the permitted use, you will need to obtain permission directly from the copyright holder. To view a copy of this licence, visit http://creativecommons.org/licenses/by/4.0/.

\section{References}

1. European commission: Flightpath 2050-Europe's vision for aviation-Report of the high level group on aviation research. Available: https://ec.europa.eu/transport/sites/transport/files/modes/air/ doc/flightpath2050.pdf (2011). Accessed 11 Feb 2021

2. Cluster of Excellence $\mathrm{SE}^{2} \mathrm{~A}$ - Sustainable and Energy Efficient Aviation. https://www.tu-braunschweig.de/en/se2a (2021). Accessed 11 Feb 2021

3. Kryter, K.D.: The meaning and measurement of perceived noise level. Noise Control 6(5), 12-27 (1960). https://doi.org/10.1121/1. 2369423

4. Fastl, H., Widmann, U.: Subjective and physical evaluation of aircraft noise. Noise Control Eng. J. 35(2), 61-63 (1990)

5. Minard, A., Boussard, P.: Signal-based indicators for predicting the effect of audible tones in the aircraft sound at takeoff. INTERNOISE and NOISE-CON Congress and conference proceedings. 256(2), 6410-6419 (2016)

6. Torija, A.J., Roberts, S., Woodward, R., Flindell, I.H., McKenzie, A.R., Self, R.H.: On the assessment of subjective response to tonal content of contemporary aircraft noise. Appl. Acous. 146, 190-203 (2019). https://doi.org/10.1016/j.apacoust.2018.11.015

7. Sahai, A. K.: Consideration of aircraft noise annoyance during conceptual aircraft design. Doctoral thesis, Institut für Luft und Raumfahrtsysteme, RWTH Aachen (2016). https://publications. rwth-aachen.de/record/668901

8. Albarrán, M.Y.P., Stumpf, E.: Aircraft engine positioning psychoacoustic optimization within conceptual aircraft design. INTERNOISE and NOISE-CON Congress and conference proceedings 259(4), 5265-5276 (2019)

9. Merino-Martinez, R., Vieira, A., Snellen, M., Simons, D. G.: Sound quality metrics applied to aircraft components under operational conditions using a microphone array. In: 25th AIAA/ CEAS aeroacoustics conference (2019). https://doi.org/10.2514/6. 2019-2513

10. Vieira, A., Snellen, M., Simons, D.G.: Experimental assessment of sound quality metrics for takeoff and landing Aircraft. AIAA J. (2020). https://doi.org/10.2514/1.J059633

11. Albarrán, M. Y. P., Schültke, F., Stumpf, E.: Sound quality assessments of over-the-wing engine configurations applied to continuous descent approaches. In: proceedings of AIAA/CEAS aeroacoustics conference (2018). https://doi.org/10.2514/6.2018-4083

12. More, S. R.: Aircraft noise characteristics and metrics. Doctoral thesis, Purdue University (2010). https://docs.lib.purdue.edu/disse rtations/AAI3453255/
13. Rizzi, S., Christian, A.: A psychoacoustic evaluation of noise signatures from advanced civil transport aircraft. In: 22nd AIAA/ CEAS Aeroacoustics conference (2016). https://doi.org/10. 2514/6.2016-2907

14. Rizzi, S., Palumbo, D., Rathsam, J., Christian, A., Rafaelof, M.: Annoyance to noise produced by a distributed electric propulsion high-lift system. In: 23rd AIAA/CEAS aeroacoustics conference (2017). https://doi.org/10.2514/6.2017-4050

15. Pieren, R., Bertsch, L., Lauper, D., Schäffer, B.: Improving future low-noise aircraft technologies using experimental perceptionbased evaluation of synthetic flyovers. Sci. Total Environ. 692, 68-81 (2019). https://doi.org/10.1016/j.scitotenv.2019.07.253

16. Boeing: Global market outlook (2016-2035). Available: http:// www.boeing.com/resources/boeingdotcom/commercial/aboutour-market/assets/downloads/cmo_print_2016_final_updated. pdf (2016). Accessed 11 Feb 2021

17. Bertsch, L.: Noise prediction within conceptual aircraft design. Doctoral thesis, Technische Universität Braunschweig, Institut für Aerodynamik und Strömungstechnik (2013). https://elib.dlr.de/ 84386/

18. Bertsch, L., Wolters, F., Heinze, W., Pott-Pollenske, M., Blinstrub, J.: System noise assessment of a tube-and-wing aircraft with geared turbofan engines. J. Aircr. 56(4), 1577-1596 (2019). https://doi.org/10.2514/1.C034935

19. Bertsch, L., Sanders, L., Thomas, R.H., Legriffon, I., June, J.C., Clark, I.A., Lorteau, M.: Comparative assessment of aircraft system noise simulation tools. J. Aircr. (2021). https://doi.org/ 10.2514/1.C036124

20. Heinze, W.: Ein Beitrag zur quantitativen Analyse der technischen und wirtschaftlichen Auslegungsgrenzen verschiedener Flugzeugkonzepte für den Transport großer Nutzlasten. ISBN:3-928628-14-3 (1994)

21. Lummer, M.: Maggi-Rubinowicz diffraction correction for raytracing calculations of engine noise shielding. In: 14th AIAA/ CEAS aeroacoustics conference (29th AIAA aeroacoustics conference) (2008). https://doi.org/10.2514/6.2008-3050

22. Bertsch, L., Clark, I. A., Thomas, R. H., Sanders, L., LeGriffon, I.: The aircraft noise simulation working group (ANSWr) - tool benchmark and reference aircraft results. In: 25th AIAA/CEAS aeroacoustics conference (2019). https://doi.org/10.2514/6. 2019-2539

23. Sanders, L., Thomas, R., Bertsch, L., LeGriffon, I., Clark, I., June, J., Lorteau, M.: The Aircraft Noise Simulation Working Group (ANSWr) - V2 aircraft results. In: 25th AIAA/CEAS aeroacoustics conference (2019). https://doi.org/10.2514/6. 2019-2540

24. Pott-Pollenske, M., Dobrzynski, W., Buchholz, H., Guerin, S., Seueressig, G., Finke, U.: Airframe noise characteristics from flyover measurements and prediction. In: 12th AIAA/CEAS aeroacoustics conference (2006). https://doi.org/10.2514/6.2006-2567

25. Pott-Pollenske, M., Dobrzynski, W., Buchholz, H., Gehlhar, B., Walle, F.: Validation of a semiempirical airframe noise prediction method through dedicated A319 flyover noise measurements. In: 8th AIAA/CEAS aeroacoustics conference and exhibit (2002). https://doi.org/10.2514/6.2002-2470

26. Rossignol, K.-S.: Development of an empirical prediction model for flap side-edge noise. In: 16th AIAA/CEAS aeroacoustics conference (2010). https://doi.org/10.2514/6.2010-3836

27. Rossignol, K.-S.: Empirical prediction of airfoil tip noise. In: 17th AIAA/CEAS aeroacoustics conference (2011). https://doi.org/10. 2514/6.2011-2733

28. Dobrzynski, W., Pott-Pollenske, M.: Slat noise source studies for farfield noise prediction. In: 7th AIAA/CEAS aeroacoustics conference (2001). https://doi.org/10.2514/6.2001-2158

29. Dobrzynski, W., Chow, L., Guion, P., Shiells, D.: A European study on landing gear airframe noise sources. In: 6th AIAA/ 
CEAS aeroacoustics conference (2000). https://doi.org/10.2514/6. 2000-1971

30. Heidmann, M.F.: Interim prediction method for fan and compressor source noise. Technical memorandum. NASA TMX-71763 (1979). https://ntrs.nasa.gov/citations/19750017876

31. Stone, J.R., Groesbeck, D.E., Zola, C.L.: Conventional profile coaxial jet noise prediction. AIAA J. 21(3), 336-342 (1983). https://doi.org/10.2514/3.8077

32. ISO 9613-2:1996: Acoustics—attenuation of sound during propagation outdoors-Part 2: a general method of calculation. International Organization for Standardization, Geneva, Switzerland (1996)

33. AIR 1751: Prediction method for lateral attenuation of airplane noise during takeoff and landing. United States Society of Automotive Engineers, SAE International (1981)

34. International civil aviation organization: environmental protection-Annex 16 to the convention on international civil aviationVolume 1-aircraft noise. ISBN: 978-92-9231-108-7

35. Rizzi, S., LeGriffon, I., Pieren, R., Bertsch, L.: A comparison of aircraft flyover auralizations by the aircraft noise simulation working group. In: AIAA AVIATION 2020 FORUM (2020). https:// doi.org/10.2514/6.2020-2582

36. ISO 532-1:2017: Acoustics-Methods for calculating loudnessPart 1: Zwicker method. International Organization for Standardization, Geneva, Switzerland (2017)

37. Zwicker, E., Fastl, H.: Psychoacoustics: Facts and Models. Springer, Berlin (1999). https://doi.org/10.1007/ 978-3-540-68888-4

38. DIN 45692:2009-08: Measurement technique for the simulation of the auditory sensation of sharpness. Deutsches Institut für Normung: Berlin, Germany (2009)

39. Aures, W.: Berechnungsverfahren für den sensorischen Wohlklang beliebiger Schallsignale. Acta Acust. United Acust. 59(2), 130-141 (1985)

40. Hasting, A., Lee, K.H., Davies, P., Surprenant, A.M.: Measurement of the attributes of complex tonal components commonly found in product sound. Noise Control Eng. J. 51(4), 195-209 (2003). https://doi.org/10.3397/1.2839715
41. Terhardt, E., Stoll, G., Seewann, M.: Algorithm for extraction of pitch and pitch salience from complex tonal signals. J. Acoust. Soc. Am. 71(3), 679-688 (1982). https://doi.org/10.1121/1. 387544

42. Lawrence, J.: Aeroacoustic interactions of installed subsonic round jets. Doctoral thesis, University of Southampton (2014). https://eprints.soton.ac.uk/367059/

43. Greco, G. F.: A computational investigation of jet-plate interaction noise using a Lattice-Boltzmann based method. Master's thesis, Federal University of Santa Catarina (2018). https://repositorio. ufsc.br/handle/123456789/205670

44. Blinstrub, J.: Immission-based noise reduction within conceptual aircraft design. Doctoral thesis, Technische Universität Braunschweig (2019). https://elib.dlr.de/127483/

45. Delfs, J., Bertsch, L., Zellmann, C., Lennart, R., Kian Far, E., Ring, T., Langer, S.C.: Aircraft noise assessment-from single components to large scenarios. Energies (2018). https://doi.org/ 10.3390/en11020429

46. Merino-Martinez, R., Snellen, M.: Implementation of tonal cavity noise estimations in landing gear noise prediction models. In: AIAA AVIATION 2020 FORUM (2020). https://doi.org/10. 2514/6.2020-2578

47. Merino-Martínez, R., Neri, E., Snellen, M., Kennedy, J., Simons, D.G., Bennett, G.J.: Multi-approach study of nose landing gear noise. Journal of Aircraft 57(3), 517-533 (2020). https://doi.org/ 10.2514/1.C035655

48. Zellmann, C., Jäger, D., Schlatter, F.: Model adjustment and validation to account for the airflow deflector retrofit of the A320 family. In: Proceedings of Euronoise 2018, pp 225-230 (2018). http:// www.euronoise2018.eu/docs/papers/38_Euronoise2018.pdf

Publisher's Note Springer Nature remains neutral with regard to jurisdictional claims in published maps and institutional affiliations. 